\title{
Space anisotropy search at colliders
}

\author{
I. S. Karpikov D. A. Tlisov², D. V. Kirpichnikov? \\ Institute for Nuclear Research of the Russian Academy of Sciences, \\ 60th October Anniversary prospect 7a, Moscow 117312, Russia
}

\begin{abstract}
In the framework of model with Lorentz violation (LV) we discuss a physical observables for $q \bar{q}$ pair production at lepton-lepton colliders and describe the experimental signal to be detected. We obtain a conservative limits on Lorentz-violating dimensionless coupling for quark sector from LEP data. We also make a phenomenological prediction for LV model at the future lepton collider.
\end{abstract}

PACS: 12.60.Cn, 13.60.Fz, 14.65.Fy Keywords: Lorentz violation, Standard Model Extension (SME), lepton colliders.

\section{Introduction}

The problem of space-time anisotropy is a great challenge of high energy physics. The attempts to measure the space anisotropy for a relatively low energy scale are widely performed by an astrophysical experimental searches. Nevertheless, the ability to search fundamental properties of space-time on a high energy scales appears with LHC launching. A violation of Lorentz invariance is one of the possible reason of space anisotropy. There are various self-consistent setups of quantum gravity which admit the violation of Lorentz invariance: a models of quantum loop gravity [1, 2], string model setups [3, 4], a models of Horava-Lifshitz with extra spatial derivatives [5, 6, 7], the models of the analogue gravity [8]. The most general Lorentz-violating Lagrangian with gauge invariant renormalizable terms was performed by [9, 10] for the particles of standard model (SM). The former framework is known as Standard-Model Extension (SME) of Alan Kosteletsky. The current constraints on SME parameters are presented in [11]. In particular, the limits for leptons have been set at the level of $10^{-6}-10^{-20}$.

A very recent result [12] claims that $C P T$-even coefficients for $\mathrm{LV}$ in the quark sector (e.g. for $u$ and $d$ quarks) can be bounded at the level about $10^{-5}-10^{-6}$ from HERA experimental data on deep inelastic scattering (DIS) of $e^{-} p$. A framework of SME has been explored carefully also in the context of Tevatron collider phenomenology. In particular Ref. [13] provides the bounds on $C P T$-even LV couplings of top quark from dependence of the $t \bar{t}$ production cross-section on sideral time as the orientation of the D0 detector changes with the rotation of the Earth. Test of $C P T$-odd symmetry violation for $B$-mesons was performed in Refs. [14, 15].

However the limits on $C P T$-even $\mathrm{LV}$ coupling $\left(c_{Q(U, D)}\right)_{Z Z A B}$ for quarks hasn't been obtained yet. In the present paper we discuss a possible implication of the SME phenomenology for quark

\footnotetext{
${ }^{1}$ e-mail: karpikov@inr.ru

${ }^{2}$ e-mail: dtlisov@cern.ch

${ }^{3}$ e-mail: kirpich@ms2.inr.ac.ru
} 
sector at a lepton- lepton colliders. Namely, we calculate the production cross-section of $q \bar{q}$ pair via $\gamma$ and $Z^{0}$ - boson for the SME couplings that affect the quark field.

The paper is organized as follows. In Sec. 2 we consider general Lagrangian of the SME and perform LV couplings of quarks to be constrained by collider experiment. In Sec. 3 we derive the matrix element squared for the process $e^{+} e^{-} \rightarrow q \bar{q}$ in SME. In Sec. 4 we consider the spatial transformations from a Sun-centered reference frame to the Earth-based laboratory frame. In Sec. 5 we obtain a very conservative limits for LV coupling of $u, d, s, c$ and $b$ quarks from ALEPH and OPAL data. In Sec. 6] we derive time-dependent cross-section for the process $e^{+} e^{-} \rightarrow q \bar{q}$ and make SME prediction for the future lepton-lepton collider.

\section{SME Lagrangian}

We begin with a general SME Lagrangian, which can be expressed in the following form

$$
\mathcal{L}_{S M E}=\mathcal{L}_{S M}+\mathcal{L}_{L V}
$$

where $\mathcal{L}_{S M}$ is the standard model (SM) Lagrangian and $\mathcal{L}_{L V}$ contains renormalizable Lorentzviolating terms for SM fields. Now let us consider CPT even Lagrangian for the quark sector

$$
\begin{gathered}
\mathcal{L}_{L V} \supset \mathcal{L}_{L V}^{\text {quarks }}=i\left(c_{Q}\right)_{\mu \nu A B} \bar{Q}_{A} \gamma^{\mu} D^{\nu} \bar{Q}_{B}+i\left(c_{U}\right)_{\mu \nu A B} \bar{U}_{A} \gamma^{\mu} D^{\nu} \bar{U}_{B}+ \\
+i\left(c_{D}\right)_{\mu \nu A B} \bar{D}_{A} \gamma^{\mu} D^{\nu} \bar{D}_{B}
\end{gathered}
$$

where index $A$ labels the quark flavor, $A=1,2,3$, here $u_{A}=(u, c, t)$ and $d_{A}=(d, s, b)$. We denote left- and right-handed quarks in (2) by $Q_{A}=\left(u_{A}, d_{A}\right)_{L}, U_{A}=\left(u_{A}\right)_{R}$ and $D_{A}=$ $\left(d_{A}\right)_{R}$. The dimensionless LV coefficients $\left(c_{Q}\right)_{\mu \nu A B},\left(c_{U}\right)_{\mu \nu A B}$ and $\left(c_{D}\right)_{\mu \nu A B}$ can be assumed symmetric in flavor indices, $A, B$ and traceless in space-time indices, $\mu, \nu$. For definiteness in the present paper we consider a very specific case of Lorentz violation instead of treating full SME Lagrangian (2) for quarks, when $A=B$. Namely, the subjects of our interest are the Lagrangians for quarks in the $S U(2) \times U(1)$ breaking sector, $\mathcal{L}_{L V}=\sum_{q}\left(\mathcal{L}_{L V}^{\gamma \bar{q} q}+\mathcal{L}_{L V}^{Z \bar{q} q}\right)$. As an illustration we perform below the lagrangian for $b$ quark

$$
\begin{gathered}
\mathcal{L}_{L V}^{\gamma \bar{b} b}=Q_{b} e \bar{b}\left(c_{Q \mu \nu} \frac{\left(1-\gamma_{5}\right)}{2}+c_{D \mu \nu} \frac{\left(1+\gamma_{5}\right)}{2}\right) \gamma^{\mu} b A^{\nu} \\
\mathcal{L}_{L V}^{Z \bar{b} b}=\frac{e}{\sin 2 \theta_{W}} \bar{b}\left(c_{Q \mu \nu} C_{L}^{f} \frac{\left(1-\gamma_{5}\right)}{2}+c_{D \mu \nu} C_{R}^{f} \frac{\left(1+\gamma_{5}\right)}{2}\right) \gamma^{\mu} b Z^{\nu}
\end{gathered}
$$

here we denote for simplicity $c_{Q \mu \nu} \equiv c_{Q \mu \nu 33}$ and $c_{D \mu \nu} \equiv c_{D \mu \nu 33}$. For other flavors only diagonal elements have been left, say, for $c$-quark we have $c_{Q \mu \nu} \equiv c_{Q \mu \nu 22}$ and $c_{U \mu \nu} \equiv c_{U \mu \nu 22}$. These coefficients to be constrained by the collider experiment. All remaining LV coefficients for quarks in (2) can be set to zero without loss of generality. We also use a convenient SM notations $C_{L}^{f}=2 T_{3}^{f}-2 Q_{f} \sin ^{2} \theta_{W}$ and $C_{R}^{f}=-2 Q_{f} \sin ^{2} \theta_{W}$ in (3) and (4). 


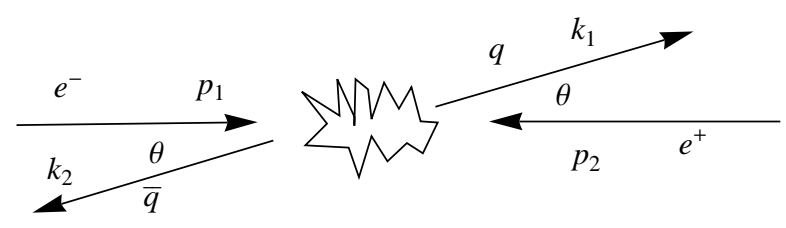

Figure 1: the diagram illustrates the kinematics of the process $e^{+} e^{-} \rightarrow q \bar{q}$.

\section{The matrix element for SME setup}

In this section we calculate the matrix element squared for the signal process $e^{+} e^{-} \rightarrow q \bar{q}$ at lepton- lepton collider for the case of Lorentz violation (3) and (4). The amplitude squared, which corresponds to $q-\bar{q}$ pair production via $\gamma$ and $Z^{0}$ boson can be written as sum of SM term and SM-SME interference terms in the leading order of $\mathrm{LV}$ couplings $c_{\mu \nu}^{Q}$, $c_{\mu \nu}^{U}$ and $c_{\mu \nu}^{D}$

$$
\overline{\sum_{\text {s.c. }}}\left|\mathcal{M}\left(e^{+} e^{-} \rightarrow q \bar{q}\right)\right|^{2} \simeq \underbrace{\overline{\sum_{s . c .}}\left|\mathcal{M}_{\gamma}+\mathcal{M}_{Z}\right|^{2}}_{|M|_{S M}^{2}}+\underbrace{\overline{\sum_{\text {s.c. }}}\left(2 \mathcal{M}_{\gamma}^{\dagger} \delta \mathcal{M}_{\gamma}+4 \mathcal{M}_{\gamma}^{\dagger} \delta \mathcal{M}_{Z}+2 \mathcal{M}_{Z}^{\dagger} \delta \mathcal{M}_{Z}\right)}_{\delta|M|_{S M E}^{2}},
$$

in the expression above we average the amplitude squared over the initial state of lepton polarization and sum over the quark colors. For the sake of simplicity we now set $c_{\mu \nu}^{Q}=c_{\mu \nu}^{U}=$ $c_{\mu \nu}^{D} \equiv c_{\mu \nu}^{q}$, then the partial amplitudes take the following forms

$$
\begin{aligned}
& \overline{\sum_{s . c .}} 2 \mathcal{M}_{Z}^{\dagger} \delta \mathcal{M}_{Z}=\frac{2 N_{c} e^{4}}{\sin ^{4} 2 \theta_{W}} \frac{c_{\mu \nu}^{q}}{\left(s-M_{Z}^{2}\right)^{2}}\left(\left(C_{L}^{q 2}+C_{R}^{q 2}\right)\left(C_{L}^{l 2}+C_{R}^{l 2}\right) L_{V}^{\mu \nu}+\left(C_{L}^{q 2}-C_{R}^{q 2}\right)\left(C_{L}^{l 2}-C_{R}^{l 2}\right) L_{A}^{\mu \nu}\right), \\
& \overline{\sum_{s . c .}} 4 \mathcal{M}_{\gamma}^{\dagger} \delta \mathcal{M}_{Z}=\frac{2 N_{c} e^{4} Q_{q} Q_{l}}{\sin ^{2} 2 \theta_{W}} \frac{c_{\mu \nu}^{q}}{s\left(s-M_{Z}^{2}\right)}\left(\left(C_{L}^{q}+C_{R}^{q}\right)\left(C_{L}^{l}+C_{R}^{l}\right) L_{V}^{\mu \nu}+\left(C_{L}^{q}-C_{R}^{q}\right)\left(C_{L}^{l}-C_{R}^{l}\right) L_{A}^{\mu \nu}\right), \\
& \overline{\sum_{s . c .}} 2 \mathcal{M}_{\gamma}^{\dagger} \delta \mathcal{M}_{\gamma}=2 N_{c} 2 e^{4} Q_{q}^{2} Q_{l}^{2} \frac{1}{s^{2}} 2 c_{\mu \nu}^{q} L_{V}^{\mu \nu},
\end{aligned}
$$

where $L_{\mu \nu}^{V}$ and $L_{\mu \nu}^{A}$ are the vector and the axial Lorentz violating tensors respectively, which depend on the 4 -momenta of the incoming and produced particles $e^{-}\left(p_{1}\right) e^{+}\left(p_{2}\right) \rightarrow q\left(k_{1}\right) \bar{q}\left(k_{2}\right)$ :

$$
\begin{gathered}
L_{\mu \nu}^{A}=\left(\left(p_{2} k_{1}\right)^{2}-\left(p_{2} k_{2}\right)^{2}\right) g_{\mu \nu}-\left(p_{2} k_{1}\right)\left(k_{2 \mu} p_{1 \nu}+k_{1 \mu} p_{2 \nu}\right)+\left(p_{2} k_{2}\right)\left(k_{1 \mu} p_{1 \nu}+k_{2 \mu} p_{2 \nu}\right), \\
L_{\mu \nu}^{V}=\left(p_{2} k_{1}\right)\left(k_{1 \nu} p_{2 \mu}+k_{2 \nu} p_{1 \mu}\right)+\left(p_{2} k_{2}\right)\left(k_{1 \nu} p_{1 \mu}+k_{2 \nu} p_{2 \mu}\right)- \\
-\left(p_{1} p_{2}\right)\left(k_{2 \mu} k_{1 \nu}+k_{1 \mu} k_{2 \nu}+p_{2 \mu} p_{1 \nu}+p_{1 \mu} p_{2 \nu}\right)+g_{\mu \nu}\left(p_{1} p_{2}\right)^{2} .
\end{gathered}
$$

In Sec. 5 and Sec. 6 we compare the matrix element (5) to the SM expectation in order to estimate the contribution of LV coefficients $c_{\mu \nu}^{q}$ to the production rate of $q-\bar{q}$ pair at the leptonlepton colliders. 

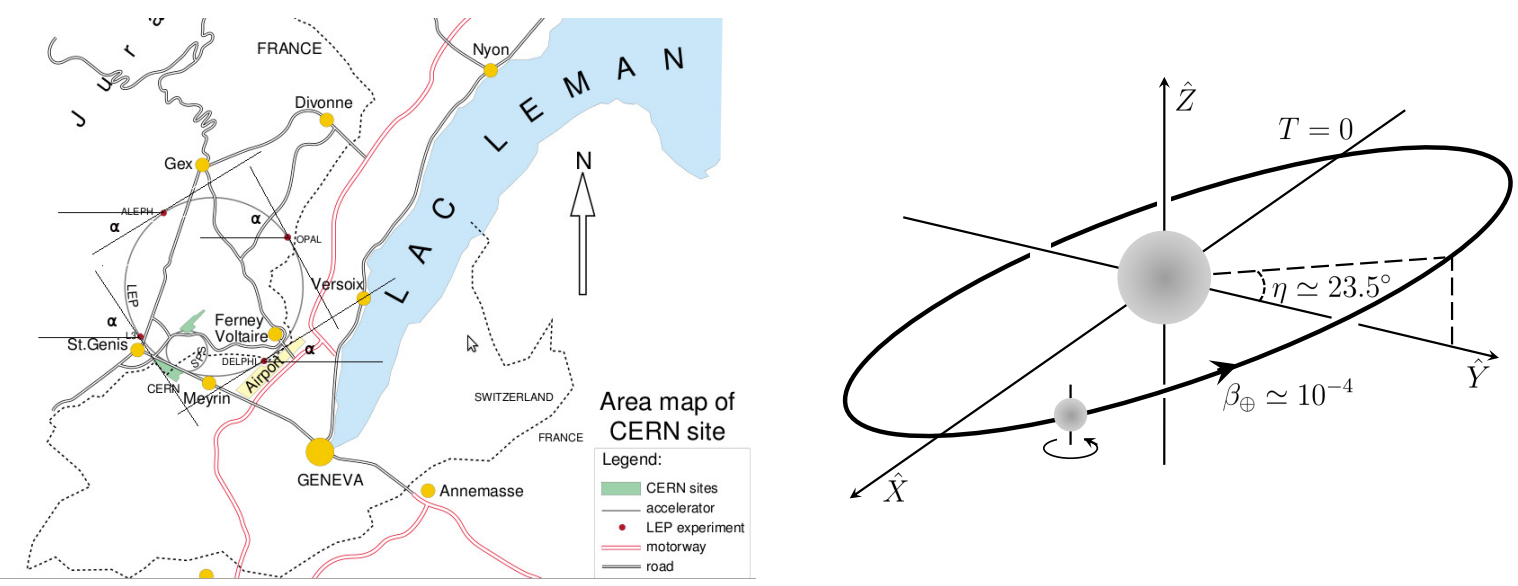

Figure 2: Left panel: orientation of the beam direction for ALEPH and OPAL detectors. Right panel: schematic illustration of the Sun-centered and Earth-based reference frames.

\begin{tabular}{|c|c|c|c|c|}
\hline & ALEPH & OPAL & L3 & DELPHI \\
\hline Beam orientation $(\alpha)$ & $33.92^{\circ}$ & $54.50^{\circ}$ & $55.60^{\circ}$ & $34.87^{\circ}$ \\
\hline Colatitude $(\chi)$ & $43.77^{\circ}$ & $43.77^{\circ}$ & $43.77^{\circ}$ & $43.77^{\circ}$ \\
\hline
\end{tabular}

Table 1: The location of the LEP detectors at Earth-based reference frame.

\section{The reference frame transformation}

If one takes into account the Earth's rotation effect, then we should replace $c_{i j}^{q} \rightarrow c_{i j}^{q}(t)=$ $c_{I J}^{q} R_{i}^{I}(t) R_{j}^{J}(t)$ in Eqs. (6-8), the indices $I$ and $J$ numerate the coordinates of the Sun-centered frame, $I, J=(X, Y, Z)$; the indices $i$ and $j$ are associated with Earth-based reference frame (see e.g. Fig. 2 for details). For the sake of simplicity we set also $c_{T T}^{q}=c_{T I}^{q}=c_{I T}^{q}=0$ throughout the paper. We assume that the relative velocity of Sun-centered and Earth-based reference frames is negligible, so the transformation operation involves only rotations. The explicit form of the rotation matrix $\hat{R}(t)=R_{i}^{J}(t)$ is given by the following partial transformations

$$
\hat{R}(t)=R_{z}(\omega t) R_{y}(\chi) R_{x}(\pi / 2) R_{y}(\alpha) .
$$

The corresponding matrices, $R_{x}(\phi) R_{y}(\theta)$ and $R_{z}(\psi)$ are defined by the following way

$R_{x}(\phi)=\left(\begin{array}{ccc}1 & 0 & 0 \\ 0 & \cos \phi & -\sin \phi \\ 0 & \sin \phi & \cos \phi\end{array}\right), R_{y}(\theta)=\left(\begin{array}{ccc}\cos \theta & 0 & -\sin \theta \\ 0 & 1 & 0 \\ \sin \theta & 0 & \cos \theta\end{array}\right), R_{z}(\psi)=\left(\begin{array}{ccc}\cos \psi & \sin \psi & 0 \\ -\sin \psi & \cos \psi & 0 \\ 0 & 0 & 1\end{array}\right)$

here $\omega=2 \pi / T_{\text {sid }}$ is related to the sideral period, $T_{\text {sid }}=(23 \mathrm{~h}, 56 \mathrm{~m}, 4.091 \mathrm{~s}), \chi$ is the colatitude of the detector, $\chi=\left(90^{\circ}\right.$-Latitude $)$, and $\alpha$ is the angle between the lepton beam and detector's longitude. One can see from Eqs. (6) that SME amplitudes squared have the terms which are proportional to the vector part, $c_{I J}^{q} R_{i}^{I}(t) R_{j}^{J}(t) L_{i j}^{V}$, and to the axial part, $c_{I J}^{q} R_{i}^{I}(t) R_{j}^{J}(t) L_{i j}^{A}$. Thus the effect of Earth's rotation will introduce a time dependence in the SME contribution, $\delta|\mathcal{M}|_{S M E}^{2}(t)$, to the production rate of $q-\bar{q}$ pair. 


\section{$5 \quad q \bar{q}$ pair production at LEP}

The differential cross-section for $q \bar{q}$ pair production at LEP including Lorentz-violating contribution from SME can be written in the following form

$$
\frac{d \sigma}{d \Omega}\left(e^{+} e^{-} \rightarrow q \bar{q}\right)=\frac{1}{64 \pi^{2} s} \overline{\sum_{s . c .}}\left(|\mathcal{M}|_{S M}^{2}+\delta|\mathcal{M}|_{S M E}^{2}(t)\right)
$$

In this section we restrict our analysis to the case

$$
c_{I J}^{q}=\left(\begin{array}{lll}
c_{X X}^{q} & c_{X Y}^{q} & c_{X Z}^{q} \\
c_{Y X}^{q} & c_{Y Y}^{q} & c_{Y Z}^{q} \\
c_{Z X}^{q} & c_{Z Y}^{q} & c_{Z Z}^{q}
\end{array}\right) .
$$

The traceless condition for $c_{I J}$ requires that $c_{X X}^{q}+c_{Y Y}^{q}=-c_{Z Z}^{q}$. In order to estimate the collider sensitivity to SME coefficient we average $\delta|M|_{S M E}^{2}(t)$ over the sideral period, $T_{\text {sid }}$. The explicit calculation revealed that time-averaged SME amplitude is proportional to SM matrix element squared

$$
\left\langle\delta|M|_{S M E}^{2}(t)\right\rangle_{t}=\frac{1}{T_{\text {sid }}} \int_{0}^{T_{\text {sid }}} \delta|M|_{S M E}^{2}(t) d t=C_{S M E} \cdot|M|_{S M}^{2}
$$

where

$$
C_{S M E}=\frac{c_{Z Z}^{q}}{8}(1+3(\cos 2 \alpha+\cos 2 \chi-\cos 2 \alpha \cos 2 \chi)) .
$$

Which means that SME coefficients contribute to the signal cross-section up to the multiplicative factor in the following way

$$
\sigma_{e^{+} e^{-} \rightarrow q \bar{q}}^{S M E}=\sigma_{e^{+} e^{-} \rightarrow q \bar{q}}^{S M} \cdot\left(1+C_{S M E}\right) .
$$

It must be point out that after time-averaging (14) our analysis is not sensitive to $X Y, X Z$ or $Z Y$ elements of (13). So we can constrain only $Z Z$ component of SME coupling. Since ALEPH and OPAL detectors at LEP measured directly the production rate of $q \bar{q}$ events, from experimental uncertainties on $\sigma_{e^{+} e^{-} \rightarrow q \bar{q}}^{S M}$ we can derive the limits on $\left|c_{Z Z}^{q}\right|$ under assumption $\left|c_{Z Z}^{u}\right|=\left|c_{Z Z}^{d}\right|=\left|c_{Z Z}^{s}\right|=\left|c_{Z Z}^{c}\right|=\left|c_{Z Z}^{b}\right|=\left|c_{Z Z}\right|$, Tab. 2 shows relevant constraints. Beyond this assumption, namely for $\left|c_{Z Z}^{u}\right| \neq\left|c_{Z Z}^{d}\right| \neq\left|c_{Z Z}^{s}\right| \neq\left|c_{Z Z}^{c}\right| \neq\left|c_{Z Z}^{b}\right|$, the systematic uncertainty of $b \bar{b}$ - and $c \bar{c}$ - pairs fraction in total $q \bar{q}$ production needs to be taken into account. It follows from $\sigma_{b \bar{b}(c \bar{c})}=R_{b(c)} \cdot \sigma_{q \bar{q}}$ that the relative uncertanties on $b \bar{b}(c \bar{c})$ cross-section can be expressed in the following way $\Delta \sigma_{b \bar{b}(c \bar{c})} /\left\langle\sigma_{b \bar{b}(c \bar{c})}\right\rangle=\Delta \sigma_{q \bar{q}} /\left\langle\sigma_{q \bar{q}}\right\rangle+\Delta R_{b(c)} /\left\langle R_{b(c)}\right\rangle$. In this case conservative bounds can be found in Tab. 3 for ALEPH and OPAL detectors.

\section{The prospects of SME probes in quark sector}

In this section we briefly discuss a possible implications of the SME phenomenology for collider experiments and for low energy searches of LV. In contrast to the Sec. 5 now we consider the 


\begin{tabular}{|c|c|c|}
\hline & ALEPH & OPAL \\
\hline$\Delta \sigma_{q \bar{q}} /\left\langle\sigma_{q \bar{q}}\right\rangle$ & $0.78 \%$, see Tab. 4 of Ref. [16] & $1.21 \%$, see Tab. 5 of Ref. [18] \\
\hline$\left|c_{Z Z}\right|$ & $<0.027$ & $<0.036$ \\
\hline
\end{tabular}

Table 2: Conservative bounds on LV coupling of all quarks assuming $\left|c_{Z Z}^{u}\right|=\left|c_{Z Z}^{d}\right|=\left|c_{Z Z}^{s}\right|=$ $\left|c_{Z Z}^{c}\right|=\left|c_{Z Z}^{b}\right|=\left|c_{Z Z}\right|$.

\begin{tabular}{|c|c|c|}
\hline & ALEPH & OPAL \\
\hline$\Delta \sigma_{q \bar{q}} /\left\langle\sigma_{q \bar{q}}\right\rangle$ & $0.78 \%$, see Tab. 4 of Ref. [16] & $2.2 \%$, see Tab. 2 of Ref.[19] \\
\hline$\Delta R_{b} /\left\langle R_{b}\right\rangle$ & $9.2 \%$, see Sec. 7.1 of Ref. [16] & $13.5 \%$, see Sec. 2.2 of Ref. [19] \\
\hline$\Delta R_{c} /\left\langle R_{c}\right\rangle$ & $10.8 \%$, see Sec. 7.2 of Ref. [16] & - \\
\hline$\left|c_{Z Z}^{b}\right|$ & $<0.35$ & $<0.46$ \\
\hline$\left|c_{Z Z}^{c}\right|$ & $<0.4$ & - \\
\hline
\end{tabular}

Table 3: Conservative bounds on LV coupling of $c$ - and $b$-quarks.

effects of time-dependence in the LV cross-section (12). In order to minimize the number of parameters to be constrained we choose the following benchmark matrix of LV dimensionless couplings

$$
c_{I J}^{q}=\left(\begin{array}{ccc}
c_{X X}^{q} & 0 & 0 \\
0 & -c_{X X}^{q} & 0 \\
0 & 0 & 0
\end{array}\right) .
$$

In this case the cross-section for $q \bar{q}$ production can be presented as the cross-section of SM process, $\sigma_{e^{+} e^{-} \rightarrow q \bar{q}}^{S M}$, modulated by a time dependent function

$$
\sigma_{e^{+} e^{-} \rightarrow q \bar{q}}^{S M E}=\sigma_{e^{+} e^{-} \rightarrow q \bar{q}}^{S M} \cdot(1+\epsilon(t)),
$$

where the contribution of LV couplings is given by the following function

$$
\epsilon(t)=c_{X X}^{q}\left(\cos 2 \omega t\left\{\cos ^{2} \alpha \cos ^{2} \chi-\sin ^{2} \alpha+\cos ^{2} \chi\right\}-\cos \chi \sin ^{2} \alpha \sin 2 \omega t\right) .
$$

The expression (17) describes the variation of $q \bar{q}$ production signal twice with the sideral day due to the Lorentz violating contribution of the SME. We leave Monte-Carlo simulation of the signal (17) for the future study.

The analyses of Refs. [12, 13] are very sophisticated and comprehensive test of SME. However, in the light of prospect study, it is instructive to probe SME for the low energy observables [20] as well as for the phenomenological quantities at the high-energy scales [21]. Indeed, Lorentz-violation in quark sector (3) affects the photon polarization operator. Moreover, one can show that Lorentz-violating kinetic term of quark Lagrangian modifies the dispersion relation of quarks at the tree level as well as the photon's dispersion relation at the one-loop level [20. This effectively means that the velocity of the photon acquires the additional contribution from the terms which "run" via renormalization-group. Therefore, one can constrain the LV coupling of quarks with a high accuracy from laser experiments by measuring the speed of light. This sophisticated analysis is a subject of our study in the nearest future. 


\section{Aknowledgments}

This work was supported by RFBR grant 16-32-00803. We thank A. Kostelecky, P. Satunin, D. Gorbunov, S. Gninenko, N. Krasnikov and V. Matveev for fruitful discussions.

\section{References}

[1] R. Gambini and J. Pullin, Phys. Rev. D 59, 124021 (1999).

[2] J. Alfaro, H. A. Morales-Tecotl and L. F. Urrutia, Phys. Rev. D 65, 103509 (2002).

[3] V. A. Kostelecky and S. Samuel, Phys. Rev. D 39, 683 (1989).

[4] V. A. Kostelecky and R. Potting, Nucl. Phys. B 359, 545 (1991).

[5] P. Horava, Phys. Rev. D 79, 084008 (2009).

[6] D. Blas, O. Pujolas and S. Sibiryakov, Phys. Rev. Lett. 104, 181302 (2010).

[7] S. Liberati and L. Maccione, Ann. Rev. Nucl. Part. Sci. 59, 245 (2009).

[8] S. Fagnocchi, S. Finazzi, S. Liberati, M. Kormos and A. Trombettoni, New J. Phys. 12, $095012(2010)$.

[9] D. Colladay and V. A. Kostelecky, Phys. Rev. D 58, 116002 (1998).

[10] V. A. Kostelecky, Phys. Rev. D 69, 105009 (2004).

[11] V. A. Kostelecky and N. Russell, Rev. Mod. Phys. 83, 11 (2011).

[12] A. Kostelecky, E. Lunghi and A. R. Vieira, arXiv:1610.08755 [hep-ph].

[13] V. M. Abazov et al. [D0 Collaboration], Phys. Rev. Lett. 108, 261603 (2012).

[14] K. R. Schubert, arXiv:1607.05882 [hep-ph].

[15] R. Aaij et al. [LHCb Collaboration], Phys. Rev. Lett. 116, no. 24, 241601 (2016) arXiv:1603.04804 [hep-ex]].

[16] S. Schael et al. [ALEPH Collaboration], Eur. Phys. J. C 49 (2007) 411.

[17] R. Barate et al. [ALEPH Collaboration], Eur. Phys. J. C 12, 183 (2000) doi:10.1007/s100529900223 hep-ex/9904011.

[18] G. Abbiendi et al. [OPAL Collaboration], Eur. Phys. J. C 33 (2004) 173.

[19] G. Abbiendi et al. [OPAL Collaboration], Eur. Phys. J. C 6, 1 (1999) doi:10.1007/s100520050318, 10.1007/s100529801027 hep-ex/9808023.

[20] P. Satunin, arXiv:1705.07796 [hep-th].

[21] M. S. Berger, V. A. Kostelecky and Z. Liu, Phys. Rev. D 93, no. 3, 036005 (2016) 